\title{
Weak spatial constancy in touch
}

\author{
Mark Wexler* \\ CNRS \& Université Paris Descartes
}

\author{
Vincent Hayward ${ }^{\dagger}$ \\ Université Pierre et Marie Curie
}

\begin{abstract}
We propose extending the concept of spatial constancy to haptic perception. In vision, spatial constancy refers to the conversion of retinotopic signals into spatiotopic representations, allowing the observer to perceive space independently of his or her own eye movements, or at least partly so. The problem would seem at least as important in haptic perception, where sensory surfaces undergo even more complex movements in space. Here we develop a methodology for studying haptic spatial constancy, which involves a tactile display mounted on a mobile platform, and which allows us to decouple movements of the sensory surface - in this case the fingertip - from movements of objects on the fingertip. Using this apparatus, we find evidence for only weak haptic spatial constancy.
\end{abstract}

\section{INTRODUCTION}

Any perceptual system with mobile sensors faces the problem of converting signals into a spatiotopic reference frame, in which objects' positions and motions are represented independently of the sensor movements and the shifts that they induce. In vision, these processes and their perceptual outcome are known as spatial constancy, and have long been an object of study by experimental psychologists, psychophysicists, and neuroscientists (see ref. [8] for a recent review). Although positions of objects abruptly change on the retina due to fast eye movements (saccades), and velocities are distorted due to slow movements (smooth pursuit), we usually perceive a stable, spatiotopic visual world, despite the retinotopic havoc caused by eye movements. However, slow eye movements may lead to less spatial constancy in vision than fast movements [5].

Despite the fact that the same problem exists for touch-and is probably more severe, due to the many more degrees of freedom that the touch sensors have-little is known about spatial constancy in the touch modality. After all, most objects that we actively touch are themselves stationary (with respect an earth-fixed reference frame), and we usually perceive them as such. Do we simply assume that objects that we actively touch as stationary, or do we calculate their motion in a spatiotopic reference frame by adding their motion on the fingertip (say) to the motion of the fingertip in space?

The goal of the present experiment is to begin to answer this question by decoupling and simultaneously varying the velocity of a simulated object on a fingertip, and the velocity of the fingertip in space, both in one dimension. The subject's task was to report the direction, left or right, of the perceived motion of the object in space. If subjects have haptic spatial constancy, their responses should depend on the sum of the two velocities (which is the actual velocity in space), and if they do not, then the responses should depend only on the velocity of the object on the fingertip.

*e-mail: mark.wexler@parisdescartes.fr

†e-mail: vincent.hayward@isir.upmc.fr

Proc. of the 2011 WorlHaptics Conference, pp. 605-607.
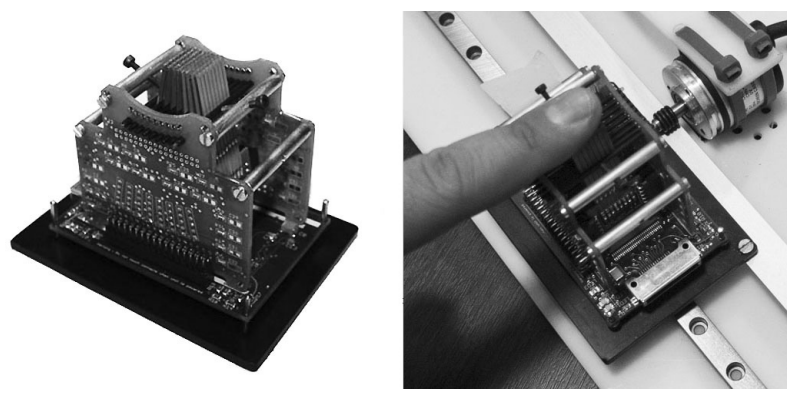

Figure 1: The Latero tactile display, shown separately (left), and mounted on the slider, beneath a fingertip (right). The slider is moved by the subject, and its position is read by means of a rotary encoder (shown on the right). The signal is sent to an Arduino microcontroller board, which activates the 64 pins of the tactile display as a function of the slider's position and the parameters of a given trial. The overall refresh rate is about $1000 \mathrm{~Hz}$.

\section{EXPERIMENT}

\subsection{Methods}

We used a tactile display called the Latero (Tactile Labs, tactilelabs.com) that operates by deforming the fingerpad skin with an array of laterally moving pins actuated by miniature piezoelectric bending motors [7]. The display consists of an $8 \times 8$ array of independent pins within an area of about $1 \mathrm{~cm}^{2}$-see Figure 1 . The Latero was mounted so as to slide on a linear track (parallel to the pins' motion), and its position was read using an optic encoder whose pulses were counted using a dedicated quadrature counter; the positional resolution was better than $10 \mu \mathrm{m}$. This position output was fed into an Arduino Mega microcontroller board (http://arduino.cc) which into turn updated the pins of the tactile display using a parallel interface, and handled infrequent serial communication with a $\mathrm{PC}$ that controlled the experiment, provided instructions to the subject, collected responses and recorded data. This setup allowed us to update the tactile display at about $1000 \mathrm{~Hz}$.

Throughout the experiment, the subject placed the pad of the index finger of his or her dominant hand on the horizontal surface of the tactile display, with the finger perpendicular to the track. The subject was seated with the elbow flexed at about $90^{\circ}$, and the track oriented so that motion could be naturally performed by rotating the elbow while keeping the wrist stiff. There was little slip between the finger pad and the display during normal sliding motion, due to the low friction of the track.

On each trial, the subject was first instructed to slide the tactile display to the left of the track. The subject then slid the display to the right, at a speed as constant as possible. During this phase, the tactile stimulus was applied. The stimulus consisted of a wave of lateral pin deflection simulating a quartic bell-shaped bump perpendicular to the $x$ dimension (which was parallel to the track), with all 8 pins in each column set to the same deflection. The deflection followed the first derivative of this shape, and therefore had the form $\chi\left(\chi^{2}-1\right)$, with $\chi=x / w$, with $w=2 \mathrm{~mm}$. Each pin could be set to one of 144 discrete levels, which was used to antialias the virtual object shape. 


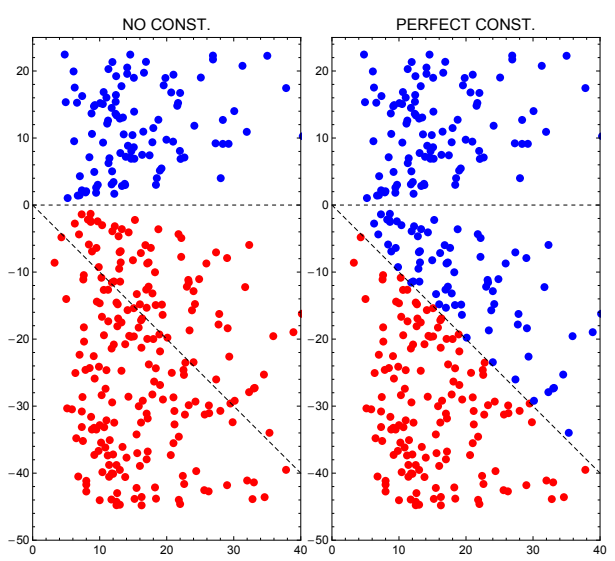

(a)
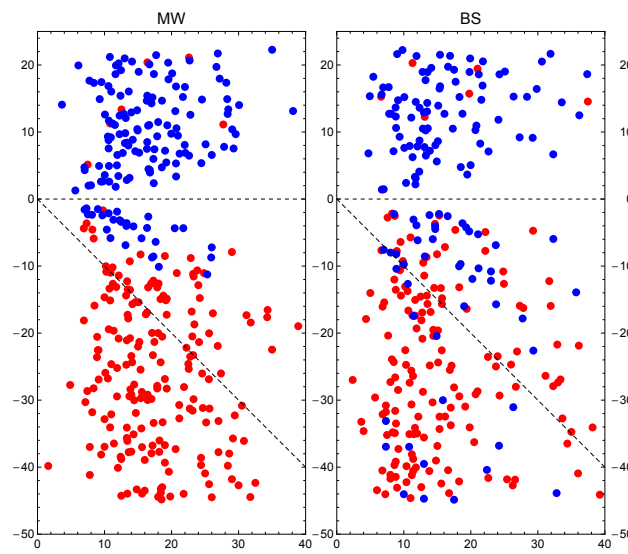

(b)

Figure 2: Ideal and real data. Each point represents one trial, with the $x$-axis representing the velocity of the finger in space, and the $y$-axis the velocity of the object with respect to the finger. Trials in which subjects reported motion to the right will be marked blue, those in which they reported motion to the left red. (a) Ideal data for no spatial constancy, and perfect constancy. (b) Real data for the three subjects.

The velocity of the virtual object, with respect to the display and therefore with respect to the finger, was randomly chosen on each trial between $-45 \mathrm{~cm} / \mathrm{s}$ (opposite to the rightward finger motion) and $+22.5 \mathrm{~cm} / \mathrm{s}$ (in the same direction as the finger). As soon as the finger crossed a threshold (randomly chosen to be with $4 \mathrm{~cm}$ of the track's center), the stimulus was virtually placed at $1 \mathrm{~cm}$ from the display's center (and therefore wasn't yet felt, since it fell outside the physical display), either to the left or to the right, depending on its subsequent motion direction. The stimulus then moved at its constant, previously chosen speed, simulating a narrow bump moving across the finger pad. When the subject's finger arrived at the far right of the track, the subject responded as to whether the felt object moved to the right or to the left in space. The reference frame was emphasized by telling the subject not to respond to motion relating to the finger, but rather relative to the table or the room. The response was given by pressing one of two mouse buttons.

We wanted subjects to spontaneously move their fingers between 5 and $30 \mathrm{~cm} / \mathrm{s}$, and to vary their speed from trial to trial within this range. We helped them to do so by giving a speed instruction at the beginning of each trial, according to which they were to either move faster or slower than on the previous trial. If the measured speed on the previous trial (during the phase when the stimulus could be felt) was between 8.75 and $26.25 \mathrm{~cm} / \mathrm{s}$, then the instruction was the same as on the previous trial. If the speed on the previous trial was either above or below this range, the subject's instruction on the next trial was to go slower or faster, respectively.

Three subjects participated in the experiment, the first author and two naïve but psychophysically experienced subjects. Each subject performed between 300 and 400 trials, which took about half an hour. The experiment was performed in a dimly lit room.

\subsection{Results}

We will represent raw data from each trial as points on a scatterplot, with the $x$-axis representing the velocity of the finger in space, and the $y$-axis the velocity of the object with respect to the finger. Color codes response: trials in which subjects reported motion to the right will be marked blue, those in which they reported motion to the left red. Ideal data, for total lack of spatial constancy, and for perfect constancy, are shown in Figure 2a. When constancy is lacking, responses are based on proximate velocity, i.e., the velocity of the object on the finger. For perfect constancy, responses are based on true spatial velocity, which is the sum of the velocity of the object on the finger and the velocity of the finger in space.

\begin{tabular}{lccc} 
Subj. & Coeff. & Lower bound & Upper bound \\
\hline MW & 0.31 & -0.07 & 0.45 \\
BS & 0.40 & -0.22 & 0.77 \\
EH & 0.10 & -0.40 & 0.52
\end{tabular}

Table 1: The fitted degree of constancy $(\kappa)$. Results are given for the three subjects, together with $95 \%$ confidence intervals.

The actual data for the three subjects are shown in Figure $2 b$. Although visually we can see some evidence for constancy, especially in the first two subjects, this appears to be much weaker than the perfect constancy shown in Figure 2a. The degree of constancy can be quantified using a linear model that assumes that perceived velocity $v_{p}$ is given in terms of velocity of the object with respect to the finger, $v_{t}$, and the velocity of the finger in space, $v_{f}$, as

$$
v_{p}=v_{t}+\kappa v_{f}
$$

where $\kappa$ is a coefficient that quantifies spatial constancy: $\kappa=0$ corresponds to no constancy, and $\kappa=1$ to perfect constancy. Subjects' responses were fitted using least-squares to

$$
R \approx \sigma\left[a\left(v_{t}+\kappa v_{f}\right)+b\right]
$$

where $\sigma$ is the logistic function. Finally, a bootstrap analysis [3] with 1000 resamples was used to calculate $95 \%$ confidence intervals on the values of the constancy coefficient $\kappa$.

The results of this analysis can be seen in Table 1, separately for the three subjects. They confirm our impression that haptic spatial constancy, if it exists at all, is extremely imperfect. In fact, we cannot statistically exclude a total lack of constancy $(\kappa=0)$ for any of the subjects. We can, however, exclude perfect constancy $(\kappa=1)$ for all of them.

\section{Discussion}

Having proposed a notion of haptic spatial constancy, we have devised a technique to measure the degree of such constancy. We have found this degree to be quite low, between $10 \%$ and $40 \%$ in our subjects. Statistically, we cannot exclude a complete lack of constancy in any of the subjects, but we can exclude perfect constancy in all of them. It is possible that the small (and statistically insignificant) degree of constancy measured in all subjects was due to explicit 
reasoning of the type, "I'm moving very fast to the right, the stimulus is moving very slowly to the left on hy fingertip, so it must be moving to the right." This sort of extra-perceptual process cannot be excluded, especially because the task was felt by all subjects as being quite hard.

We have obtained a comparable result in a different experiment recently carried out in our laboratory [4]. We studied the perception of the size of objects through active touch, reasoning that to accurately judge the size of all but the smallest objects, an observer touches one edge and then another; to determine the size of the object - the distance between the two edges touched-one either has to use proprioceptive information about the positions of the two touches, or integrate finger velocity over the period between the two touches. Using a force-feedback device (rather than a tactile display), we applied two small lateral force pulses to the moving finger, which were shown in ref. [6] to be perceived as the edges of an object. We found that most subjects are very inaccurate at judging size when it is co-varied with finger speed, and in fact have the following curious illusion: when moving twice as fast over the same object, subjects judge the object as being nearly twice as small. Thus, size judgements appear to be based on duration between touching an object's edges, rather than an integral of velocity over the duration. This last result can also been seen as evidence for a lack of spatial constancy. Together, the two results add up to a convincing argument that spatial constancy is weak, and certainly much weaker than in many cases in vision.

Additional recent evidence for weak spatial constancy in touch is provided in ref. [9], who found that under certain conditions participants failed to detect that Braille dots had changed spatial location as they moved between their fingers.

Could our results be due to the poverty of our stimulus? After all, the tactile display that we used produces lateral skin deformations, but no net lateral forces nor other geometrical cues. Evidence that our display produces stimuli perceptually similar to real objects was provided in ref. [7], where it was shown that subjects could judge of the orientation of slanted lines with an accuracy better than 10 degrees, which is in line with known thresholds in humans [1]. Moreover, the display readily produces phenomena such as tactile apparent motion [2]. However, we cannot completely exclude that something about our moving tactile stimuli affected the reference frame in which they are processed; a final confirmation of the generality of our findings will have to wait until they are reproduced with real moving objects, or with tactile displays with very different properties.

The weakness or lack of haptic spatial constancy has interesting consequences, both theoretical and practical. From the theoretical standpoint, we may ask why objects don't seem to move backwards when we run our fingers over them. After all, in the experiment described here, subjects often responded that stationary objects (ones whose velocity on the finger was equal-and-opposite to the finger's velocity in space) felt that they moved in the direction opposite to that of the finger. Why aren't we plagued with this illusion in everyday life? Could it be because we're simply not paying attention to the backward motion? If so, can some aspects of spatial constancy in vision also be explained by inattention? Another interesting possibility is that haptic spatial constancy may rely on visual spatial constancy, and may thus be intrinsically multimodal. From the practical standpoint, the weakness of spatial constancy may be useful to those who are trying to build realistic haptic interfaces, as it shows that precise compensation for the user's motion in order to simulate stationary objects may be neither necessary nor even desirable.

\section{ACKNOWLEDGEMENTS}

Supported by ERC Advanced Grant PATCH

\section{REFERENCES}

[1] R. Boven and K. Johnson. The limit of tactile spatial resolution in humans: grating orientation discrimination at the lip, tongue, and finger. Neurology, 44:2361-2366, 1994.

[2] O. Carter, T. Konkle, Q. Wang, V. Hayward, and C. Moore. Tactile rivalry demonstrated with an ambiguous apparent-motion quartet. Current Biology, 18:1050-1054, 2008.

[3] B. Efron and R. Tibshirani. An introduction to the bootstrap. Chapman and Hall, 1992.

[4] I. Fasiello, V. Hayward, G. Campion, and M. Wexler. A haptic size illusion and its near-absence in the blind. Under revision, 2011.

[5] C. Morvan and M. Wexler. The nonlinear structure of motion perception during smooth eye movements. Journal of Vision, 9:1-13, 2009

[6] G. Robles-De-La-Torre and V. Hayward. Force can overcome object geometry in the perception of shape through active touch. Nature, 412:444-448, 2001.

[7] Q. Wang and V. Hayward. Biomechanically optimized distributed tactile transducer based on lateral skin deformation. International Journal of Robotics Research, 29(4):323-335, 2010.

[8] R. H. Wurtz. Neuronal mechanisms of visual stability. Vision Research, 48(20):2070-2089, 2008.

[9] M. Ziat, V. Hayward, C. Chapman, M. Ernst, and C. Lenay. Tactile suppression of displacement. Experimental Brain Research, 206:299 $310,2010$. 